\title{
Angiogenesis inhibitors and hypoxia
}

To the editor:

In her News and Views ${ }^{1}$, Steeg called into question "the simple, elegant idea of stopping tumors with angiogenesis inhibitors," because of her presumption that "such inhibitors, by depriving tumors of oxygen, could have an unintended effect: promotion of metastasis." This opinion is based on the insightful, but misinterpreted, data in a recent report by Pennacchietti et al. ${ }^{2}$, who showed that hypoxia promotes invasive growth of tumor cells by transcriptional activation of the met proto-oncogene. From these data, Pennacchietti et al. speculated that angiogenesis inhibitors could increase tumor invasion and spread. In another News and Views, Botarro and Liotta made a similar speculation ${ }^{3}$.

The preponderance of preclinical and clinical evidence contradicts this perception, however. First, the formation of metastases is suppressed by a variety of angiogenesis inhibitors in animal models $s^{4,5}$ including VEGF antagonists ${ }^{4,5}$, TNP-470 (ref. 6), endostatin ${ }^{7}$ and interferon- $\alpha^{8}$. Second, even in the rare cases where an angiogenesis inhibitor seems to 'stimulate' formation of metastases from a primary tumor ${ }^{9}$, this effect was subsequently explained by regression of the primary tumor, resulting in loss of an angiogenesis inhibitor generated by that tumor. This is similar to the rapid appearance of metastases after certain types of primary tumors are surgically removed ${ }^{10}$ or caused to regress by ionizing radiation, resulting in decreased circulating levels of angiostatin ${ }^{11}$. Third, an increase in metastases has not been observed in large clinical trials of angiogenesis inhibitors. For example, in a recent randomized, placebo-controlled, blinded clinical trial of more than 900 patients with colorectal cancer, disease progression-defined as the growth of tumors or the emergence of new metastases-was significantly prolonged by the addition of the VEGF inhibitor Avastin to conventional chemotherapy (H. Hurwitz, personal communication). In addition, there has been no evidence of promotion of metastases in the thousands of patients treated to date with thalidomide ${ }^{12}$, interferon- $\alpha$ or cyclooxygenase- 2 inhibitors ${ }^{13}$, compounds that have a range of antiangiogenic activities in different tumors.
Steeg also emphasizes that p53-null tumor cells are relatively more resistant to hypoxia than are tumor cells with wildtype $\mathrm{p} 53$, as reported by Yu et al. ${ }^{14}$ Nevertheless, it has been shown that antiangiogenic therapy can successfully inhibit the growth of p53-null tumors, as we previously discussed ${ }^{15}$. Hypoxia in a tumor induces a number of compensatory mechanisms, including the induction of proangiogenic genes through the hypoxiainducible factor (HIF)-l $\alpha$ pathway and potential increases in tumor cell invasiveness and that these mechanisms merit further investigation. Some have assumed that these compensatory pathways would be the Achilles' heel of antiangiogenic therapy. But in fact, antiangiogenic therapy in preclinical and clinical studies continues to demonstrate that growth of primary tumors and their metastases can be inhibited in the presence of activation of proangiogenic pathways. Angiogenesis inhibitors do far more than simply "deprive a tumor of oxygen.” They inhibit vascular leakage in a tumor, inhibit endothelial nitric oxide synthase, downregulate expression of HIF$1 \alpha$, upregulate expression of an inhibitor of HIF-l $\alpha$, upregulate thrombospondin (a potent endogenous angiogenesis inhibitor) and downregulate plasmin production, to name just a few activities ${ }^{16,17}$. In other words, certain angiogenesis inhibitors can counteract the compensatory pathways that tumors use in response to hypoxia. Taken together, these reports provide strong evidence that the use of angiogenesis inhibitors, alone or in combinations, does not promote, and can in fact inhibit, metastasis. This evidence supports the clinical testing of angiogenesis inhibitors for the treatment of both primary tumors and metastases.

COMPETING INTERESTS STATEMENT

The authors declare that they have no competing financial interests.

\author{
Mark W. Kieran ${ }^{1}$, Judah Folkman ${ }^{2}$ \& \\ John Heymach ${ }^{3}$ \\ ${ }^{1}$ Department of Pediatric \\ Hematology/Oncology and ${ }^{2}$ Department of \\ Surgery, The Children's Hospital, 300 \\ Longwood Avenue, Boston, Massachusetts \\ 02115, USA. ${ }^{3}$ Adult Oncology, Dana-Farber \\ Cancer Institute, 44 Binney Street, Boston, \\ Massachusetts 02115, USA.
}

\section{Steeg replies:}

I appreciate the thoughtful comments of Folkman, Kieran and Heymach. The landmark work of Folkman and his colleagues in the field of antiangiogenesis has, of course, revolutionized how we all think about cancer and has provided the foundation for a generation of scientists to think about new ways to approach therapy and prevention of this disease. The extraordinary recent report ${ }^{12}$ of an antiangiogenic approach providing a survival advantage of several months is testament, in itself, of the tangible merit of this approach.

My News and Views discussed a paper linking low oxygen supply and induction of invasion. These newer findings may also merit consideration. It is the discussion of potential wrinkles in data that propels us to think about new ideas, such as combinations of antiangiogenic and anti-invasive agents. Such new ideas could turn the recent survival advantage in the colorectal carcinoma trial from several months to several years. It has, of course, been the remarkable work of Folkman and his colleagues that has inspired us all and given us the opportunity to address these interesting issues.

\section{Patricia S. Steeg}

Laboratory of Pathology, Women's Cancer Section, National Cancer Institute, National Institutes of Health, Bethesda, Maryland 20892,USA.e-mail: steegp@mail.nih.gov

1. Steeg, P.S. Angiogenesis inhibitors: motivators of metastasis? Nat. Med. 9, 822-823 (2003).

2. Pennacchietti, S. et al. Hypoxia promotes invasive growth by transcriptional activation of the met protooncogene. Cancer Cell 3, 347-361 (2003).

3. Bottaro, D.P. \& Liotta, L.A. Out of air is not out of action. Nature 423, 593-595 (2003).

4. Shaheen, R.M. et al. Antiangiogenic therapy targeting the tyrosine kinase receptor for vascular endothelial growth factor receptor inhibits the growth of colon cancer liver metastases and induces tumor and endothelial cell apoptosis. Cancer Res. 59, 5412-5416 (1999).

5. Drevs, J. et al. Effects of PTK787/ZK222584, a specific inhibitor of vascular endothelial growth factor tyrosine kinases, on primary tumor metastasis, vessel density, and blood flow in a murine renal cell carcinoma model. Cancer Res. 60, 4819-4824 (2000).

6. Inoue, K. et al. Frequent administration of angiogenesis inhibitor TNP-470 (AGM-1470) at an optimal biological dose inhibits tumor growth and metastasis of metastatic human transitional cell carcinoma in the urinary bladder. Clin. Cancer Res. 8, 2389-2398 (2002).

7. Sauter, B.V. et al. Adenovirus-mediated gene transfer of endostatin in vivo results in high level of transgene expression and inhibition of tumor growth and metastases. Proc. NatI. Acad. Sci. USA 


\section{CORRESPONDENCE}

97, 4802-4807 (2002).

8. Wang, L. et al. Mechanism of interferon alpha on inhibition of metastasis and angiogenesis of hepatocellular carcinoma after curative resection in nude mice. J. Gastrointest. Surg. 7, 587-594 (2003).

9. Kaban, L. et al. Antiangiogenic therapy with interferon alpha for giant cell lesions of the jaws. J. Oral Maxillofac. Surg. 60, 1103-1111 (2002).

10. Holmgren, L., O'Reilly, M.S. \& Folkman, J. Dormancy of micrometastases: balanced proliferation and apoptosis in the presence of angiogenesis suppression. Nat. Med. 1, 149-153 (1995).

11. Camphausen, K. et al. Radiation therapy to a primary tumor accelerates metastatic growth in mice. Cancer Res. 61, 2207-2211 (2001).

12. Weber, D. et al. Thalidomide alone or with dexamethasone for previously untreated multiple myeloma. J. Clin. Oncol. 21, 16-19 (2003).

13. Steinbach, G. et al. The effect of celecoxib, a cyclooxygenase-2 inhibitor, in familial adenomatous polyposis. N. Engl. J. Med. 342, 1946-1952 (2000).

14. Yu, J., Rak, J., Coomber, B., Hicklin, D. \& Kerbel,
R. Effect of tumor response to p53 status on tumor response to antiangiogenic therapy. Science $\mathbf{2 9 5}$, 1526-1528 (2002).

15. Browder T. et al. Antiangiogenic therapy and $\mathrm{p} 53$. Science 297, 471 (2002).

16. Mabjeesh, N.J. et al. 2ME2 inhibits tumor growth and angiogenesis by disrupting microtubules and dysregulating HIF. Cancer Cell 3, 363-375 (2003).

17. Folkman, J. \& Kalluri, R. Tumor angiogenesis. in Cancer Medicine, 6th edn. (eds. Kufe, D.W. et al.) 161-194 (B.C. Decker, Hamilton, Ontario, 2003). 